\title{
Manual de los Reglamentos del Agua en Florida: Actividades en los Pantanos y Cuencas ${ }^{1}$
}

\author{
Michael T. Olexa, Luke D'Isernia, Laura Minton, Dulcy Miller y Sara Corbett ${ }^{2}$
}

\section{Prefacio}

Este manual esta diseñado para proporcionar un resúmen autorizado, exacto y actual de las principales leyes Federales y de Florida que están directa o indirectamente relacionadas a la agricultura. Este manual debe proveer una vista general de los muchos derechos y responsabilidades que tienen los agricultores y propietarios de tierras agrícolas bajo las leyes tanto Federal como de Florida, así como también la información de los contactos apropiados para obtenerla con más detalle. Sin embargo, el lector debe estar advertido de que algunas partes de esta publicación podrían volverse obsoletas en cualquier momento, debido a que las leyes, reglas administrativas, y decisiones de la corte, sobre las cuales se basa este manual, se encuentran bajo revisión constante. Algunos detalles de las leyes citadas no se mencionan, debido a limitaciones de espacio.

Este manual es distribuido con la aclaración de que los autores no intentan proporcionar una asesoría legal o profesional, y que la información contenida aquí no debe ser considerada como un sustituto de asesoría profesional. En este manual, no se incluye toda la información para llevar a cabo el cumplimiento de las leyes Federales y de Florida y los reglamentos que rigen la protección del agua. Por estas razones, el uso de estos materiales por cualquier persona, constituyen un acuerdo para mantener libre de perjuicios a los autores, al Servicio de Extensión Cooperativa de Florida, al Instituto de los Alimentos y Ciencias Agrícolas y a la Universidad de Florida por reclamos de responsabilidad, daños o gastos provenientes de quien sea, por haberse referido o basado en la información contenida en este manual.

\section{¿Quién Regula los Pantanos?}

\section{Cuerpo de Ingenieros del Ejercito}

La protección federal de los pantanos proviene de diversas fuentes. Bajo la Ley del Agua Limpia, el Cuerpo de Ingenieros del Ejercito (CIE) está autorizado para emitir permisos para la descarga de

1. Este es el documento EDIS FE089, una publicación de Food and Resource Economics Department, Florida Cooperative Extension Service, Institute of Food and Agricultural Sciences, University of Florida, Gainesville, FL. Publicado Noviembre 2006. Por favor visite la página electrónica de EDIS en http://edis.ifas.ufl.edu.

2. Michael T. Olexa, profesor, Food and Resource Economics Department y director, Agricultural Law Center, University of Florida, Gainesville, FL, y presidente, Agricultural Law Committee, The Florida Bar; Luke D'Isernia, alumni, Levin College of Law, University of Florida, Gainesville, FL; Laura Minton, abogado, Dean, Mead, Egerton, Bloodworth, Capouano y Bozarth, PA, Orlando FL; Dulcy Miller, abogado, Foley and Lardner, LLP, Orlando, FL; y Sarah Corbett, abogado, Florida Second District Court of Appeal, Lakeland, FL. La traducción del ingles al español estuvo a cargo de Filiberto Reyes-Villanueva.

EI Instituto de Alimentos y Ciencias Agrícolas es un empleador que opera bajo Acción Afirmativa y provee Oportunidades Igualitarias, autorizado a proveer investigación, información educativa y otros servicios, únicamente a los individuos e instituciones que operan sin discriminación alguna con relación al credo, color, religión, edad, incapacidad, sexo, orientación sexual, estado civil, nacionalidad, opinion política o afiliaciones. Para más información sobre como obtener otras publicaciones de extensión, comuníquese con la oficina de Servicio de Extensión de su condado. Servicio de Extensión de la Florida / Instituto de Alimentos y Ciencias Agrícolas / Universidad de la Florida / Larry Arrington, Decano. 
material de dragado o relleno en las aguas de los Estados Unidos. Además de esta autoridad, y según la Ley Nacional de las Políticas del Medio Ambiente (LAPMA), el CIE debe completar un "estudio de impacto ambiental" antes de emitir un permiso para trabajar en áreas sensibles, especialmente en los pantanos. Como una revisión adicional sobre la autoridad de los CIEs para emitir permisos de dragado y relleno, el CIE requiere consultar con otras agencias federales y estatales siempre que sea relevante y, que por virtud de la Ley del Agua Limpia, el Administrador de la Agencia de Protección Ambiental (APA) se retenga el poder de veto de la emisión del permiso por el CIE, a la discreción de las APAs. Mientras este veto sea raramente usado, esto crea un tipo de sistema de poder-compartido.

\section{Departamento de Protección Ambiental de Florida}

A nivel estatal, el Departamento de Protección Ambiental (DPA) es el principal responsable de la protección de los pantanos. El DPA ha tenido autoridad para proteger las áreas de los pantanos ambientalmente-sensibles y áreas designadas críticas de los estados concernientes. Ejemplos más prominentes incluyen los Cayos de la Florida, el Área de los Grandes Cipreses, el Área de los Pantanos Verdes, el Área de Protección de los Everglades y el Área de la Bahía de Apalachicola.

Además, el distrito apropiado del manejo del agua (DMA) deberá ser consultado con respecto a cualquier trabajo hecho cerca de los pantanos, si era propiedad individual o no. Los DMAs han adoptado las reglas de MAAs que regulan las actividades en los pantanos bajo la supervisión del DPA. También, los DMAs están autorizados para establecer criterios específicos de permisos para operaciones de dragado y relleno en pantanos conectados o aislados.

\section{¿Qué Es Un Pantano?}

Para el propósito de permiso del CIE, los pantanos básicamente incluyen aquellas áreas con una prevalencia de vegetación adaptada para vivir en condiciones saturadas de suelo. Con respecto a la jurisdicción del DPA, han sido compilados detallados catálogos para determinar las características de cada suelo y planta identificados en los pantanos.

Sobre la solicitud, el DPA o el apropiado DMA emitirán declaraciones para los sitios particulares considerando si su jurisdicción se extiende a esa área. También pueden emitir determinaciones formales o informales para la ampliación de los pantanos. Esas declaraciones son válidas hasta por cinco años, mientras no cambien las condiciones físicas de la propiedad. Las definiciones de los DMA de los pantanos dependen de las características hidrológicas, vegetación y suelo de un área.

\section{¿Cuándo Son Requeridos los Permisos de Pantano?}

Los permisos de dragado y relleno son requeridos para cualquier actividad no-exenta, de acuerdo al Capitulo 403 de los Estatutos de Florida. El primer paso en obtener un permiso es asegurar al DPA (o DMA) que los estándares de calidad del agua del estado no serán violados por la actividad propuesta de dragado o relleno.

Segundo, el proyecto no puede ser contrario al interés público. Diversos factores deben ser considerados en esta determinación, incluyendo:

- El efecto sobre la salud publica, seguridad, beneficio y derechos de propiedad.

- Los efectos sobre los peces y la vida silvestre (especialmente en las especies amenazadas o en peligro de extinción).

- Los efectos adversos sobre la navegación o daño de erosión.

- Una variedad de otros factores tales como los efectos sobre la productividad marina, proyectos de naturaleza temporal o permanente, y efectos sobre los recursos históricos y arqueológicos.

En el análisis final, también son considerados los factores que mitigan la destrucción de los pantanos donde es inevitable algún daño al medio ambiente. 
La duración de los permisos es generalmente por cinco años y no exceden los diez años, a menos que el proyecto no pueda ser razonablemente completado dentro de ese tiempo. En esas excepcionales circunstancias, el DPA puede emitir 25 años de permisos. Permitiendo rangos de multas desde $\$ 400$ a $\$ 25.000$, dependiendo de la duración y de los pantanos afectados.

\section{¿Qué Regulaciones Están Exentas Para los Pantanos?}

- Federal: En la sección Federal, las actividades de cultivos normales, silvicultura, y actividades de ranchos en los pantanos están exentos de los permisos de relleno mandados por la Ley de Agua Limpia. Esta exención no se aplica para los equipos mecanizados usados en la tierra desmontada, así como se ha mantenido por representar una fuente de contaminación de punto de (ej: el relleno).

- Florida: En Florida, hablando en general, las actividades agrícolas y sistemas cerrados de manejo de agua agrícola están exentos de la regulación del pantano, siempre que las actividades sean consistentes con las actividades agrícolas. Las "actividades agrícolas" se definieron para incluir todas las actividades agrícolas y forestales necesarias, que son normales y habituales para un área en particular, siempre que tales operaciones no impidan o desvíen el flujo de la superficie de las aguas."El manejo cerrado de las aguas agrícolas" incluyen los sistemas de manejo del agua agrícola o forestal y estanques agrícolas que son permitidos según el Capitulo 373, de los Estatutos de Florida o exentos del requerimiento de permiso de los estatutos del manejo del agua. superficial (ver FE088, Manejo y

Almacenamiento de las Aguas Superficiales).

\section{¿Qué Trata Mitigación y Mitigación Bancaria?}

Los procesos de mitigación y mitigación bancaria pueden aplicarse para las actividades de pantano, de la misma manera que para el manejo y almacenamiento de las aguas superficiales (FE088).
La mitigación puede ser usada para contrarrestar los daños potenciales que resulten de las actividades en las áreas de los pantanos. El DPA y los DMA han establecido el criterio de cuando la mitigación es apropiada o deseable.

\section{¿Qué Es el Reclamo?}

El reclamo es el proceso de retornar la tierra que ha sido extraída, al más alto valor del dólar, por los cambios físicos de la tierra a un estado más usable. Bajo los estatutos de reclamación de la tierra de Florida, del Capitulo 378, de los Estatutos de Florida, en las Secciones 378.202, la tierra, incluyendo los pantanos, deben ser restaurados a una condición aceptable después de la operación de extracción. Además, las actividades de reclamo deben reunir los requerimientos del DPA y el apropiado DMA sobre el manejo del agua superficial y subterránea, incluyendo los estándares diseñados para los cuerpos de agua.

De acuerdo con esos requerimientos, se deberán asumir las prácticas de mejor manejo (PMM) para minimizar la erosión. También son prescritos por los PMM los procedimientos específicos para el tratamiento de la costa, donde los resultados de las excavaciones en la formación de un cuerpo de agua son los siguientes:

- El director ejecutivo del DMA debe ser notificado del propósito de extraer una parte de minerales, arcilla, carbón, grava, arena u otra sustancia sólida de valor comercial.

- Todas las actividades de reclamo deberán ser conducidas de tal manera que reduzcan los impactos adversos sobre los recursos de las aguas superficiales y subterráneas, vida silvestre y tierras adyacentes.

Una excepción para el requerimiento de notificación se aplica para las excavaciones reducidas a un acre o menos en un año determinado, sin exceder cinco acres sobre la vida de la mina o de la parte extraída. Una nota similar, de tierra de arcilla, minerales pesados, piedra caliza y fosfato están cubiertos separadamente por los requerimientos permitidos. 


\section{¿Qué Hay Acerca de los Manglares?}

El corte o remoción de los manglares está permitido solamente cuando se autorizan los permisos de dragado o relleno. El castigo de un recorte no autorizado o alteración de manglares, es el costo de la restauración. Para evitar un castigo, deberán obtenerse los permisos, y se puede otorgar un cambio en la exención, donde por el contrario, resulte una única e innecesaria penalidad al solicitante. Los permisos de ese tipo son emitidos por el DPA, el CIE, o el gobierno local con la participación de una autoridad delegada. El corte o remoción es permitida donde el impacto del medio ambiente es mínimo. En algunos casos, el recorte selectivo puede ser permitido para facilitar los derechos de diversión del ribereño. En otros casos, hay ciertas excepciones para los requerimientos del permiso, cuando se traten con los canales hechos por el hombre, los manglares previamente plantados y los manglares muertos. Los propietarios deberán consultar con el DPA o el DMA par ver si se aplica una de esas excepciones.

Las aplicaciones para cortar o remover manglares son transferidos a las oficinas del condado o municipales, que tienen jurisdicción sobre el área para el cual el permiso ha sido requerido. Los aplicantes deberán estar concientes que existen departamentos reguladores de los programas de drenado y relleno para asesorar a los aplicantes sobre estos asuntos.

\section{¿Cuáles Son los Castigos?}

la responsabilidad civil para el daño causado al agua de excavación, relleno o destrucción ilegal, de los pantanos. Las multas pueden correr tan alto como $\$ 10.000$ por ofensa, y la responsabilidad puede ser responsabilidad compartida (ver FE598, Reglamento Privado para discusión de responsabilidad compartida).

Se aplican sanciones criminales para la violación o contaminación mal intencionada pasando estas violaciones a ser felonías de tercer grado. Pueden ser impuestas multas de hasta $\$ 50.000$ por ofensa, y prisión de hasta por cinco años.
Además, cada día de no cumplimiento puede crear una violación separada. La indiferencia imprudente o la despreocupación de la probabilidad de daño a los recursos acuáticos del estado, es un delito menor de segundo grado con una multa de no más de $\$ 5.000$ o seis meses en prisión. El fallo para obtener el permiso, la falla de cumplir con las regulaciones o hacer falsas declaraciones a las autoridades pertinentes, son todos delitos menores de primer grado, castigables por una multa de no más de $\$ 10.000$ o seis meses en prisión.

\section{Fuente}

Capítulo 373 de los Estatutos de Florida, Secciones 473.413 al 373.403 y 403.9324 al 403.9333

\section{Información de Contacto}

Manejo de Pantanos y Cuencas (FE099, Agencias de Contacto)

- S-1 Distritos para el Manejo del Agua de Florida

- S-2 Departamento de Protección Ambiental de Florida

- L-1 Información General de APA, Región 4

\section{Agradecimientos}

Los autores agradecen al personal de las agencias estatales y federales por su tiempo y asesoría en la preparación de este manual. Los autores agradecen especialmente a Richard Budell del Office of Agricultural Water Policy of the Florida Department of Agriculture and Consumer Services por el apoyo económico para el desarrollo de esta publicación. 